\title{
THE CONTROL OF PORTING IN PLATFORM MARKETS
}

\author{
RUFUS POLLOCK \\ UNIVERSITY OF CAMBRIDGE
}

OCTOBER 7, 2008

\begin{abstract}
A sizable literature has grown up in recent years focusing on platform markets in which economies of scale combined with complementarities between a platform and its associated 'software' or 'services' can generate indirect network effects (that is positive feedback between the number of consumers using that platform and the utility of an individual consumer). In this paper we introduce a model of 'porting' in such markets where porting denotes the conversion of 'software' or 'services' developed for one platform to run on another. Focusing on the case where a dominant platform exists we investigate the impact on equilibrium and the consequences for welfare of the ability to control porting. Specifically, we show that the welfare costs associated with the "control of porting' may be more significant than those arising from pricing alone. This model and its associated results are of particular relevance because of the light they shed on debates about the motivations and effects of actions by a dominant platform owner. Recent examples of such debates include those about Microsoft's behaviour both in relation to its operating system and its media player, Apple's behaviour in relation to its DRM and iTunes platform, and Ebay's use of the cyber-trespass doctrine to prevent access to its site.
\end{abstract}

Keywords: Network Effects, Two-Sided Markets, Porting, Antitrust, Competition

JEL Classification: L15 L12 L13

Faculty of Economics, Cambridge University, Sidgwick Avenue, Cambridge, CB3 9DD. Email: rp240@cam.ac.uk or rufus@rufuspollock.org. I thank Rupert Gatti and David Newbery for guidance and many helpful discussions. I am also very grateful to Sanjeev Goyal, John Vickers, David Gill and participants at the 2007 ZEW Summer Workshop, the 2008 International Industrial Organization Conference and the 2008 CCRP workshop for a variety of helpful comments. All remaining errors are mine. 


\section{INTRODUCTION}

Several recent cases, which we discuss in more detail below, have focused economists' attention on the motivations and effects of the behaviour of a dominant firm in two-sided markets. We believe that much of this activity can usefully be interpreted in terms of efforts to control (and prevent) 'porting' - where porting denotes the conversion of a 'software' or 'service' associated with one platform to run on another platform. Building on the existing literature on two-sided markets, we develop a formal model of 'porting' and, focusing on the case where a dominant platform exists. We use this model to investigate the impact on equilibrium and the consequences for welfare of the ability to control porting. Specifically, we show that the welfare costs associated with the 'control of porting' may be more significantly more substantial than those arising from pricing alone.

For example, much of the 1998 case of US vs. Microsoft as well as more recent antitrust disputes in Europe over Microsoft's media player can be seen as related to efforts to control porting. In the 1998 case there was the alleged tying of Internet Explorer browser as well as efforts to undermine compatibility with other systems, for example, by subtly changing the Windows version of the Java Virtual Machine. ${ }^{1}$ Similarly, the media player dispute concerned the bundling of Microsoft's own Media Player 'for free' with the operating system. In both cases there has been considerable debate ${ }^{2}$ over the motivations for, and consequences of, Microsoft's behaviour, especially as to whether these sorts of activities could be described as 'tying'. ${ }^{3}$ To our mind much of this behaviour is best seen in light of efforts to control porting and thereby preserve the market power associated with the 'Applications Barrier to Entry' (as the indirect network effects were termed in that antitrust action). Unlike with traditional tying, Microsoft's actions, though obviously directly affecting competing applications (Netscape's Browser, Real Networks Audioplayer etc), were not directed at them. Rather, they were motivated by the fear that losing control of key Application Programming Interfaces (APIs) and user services would make it easier for end-user applications and services to move (port) between operating system platforms,

\footnotetext{
${ }^{1}$ See Judge Jackson in Findings of Fact in the case of United States vs. Microsoft, (Jackson, 1999). ${ }^{2}$ See, for example, Hall and Hall (2000); Davis and Murphy (2000); Fisher (2000); Bresnahan (2001); Liebowitz and Margolis (1999); Klein (2001); Gilbert and Katz (2001).

${ }^{3}$ See the works previously cited and, specifically on the tying issues, Whinston (1990); Bernheim and Whinston (1998) and the survey in Whinston (2001).
} 
which would, in turn, make it easier for consumers to switch between different platforms and thereby reduce Microsoft's market power. ${ }^{4}$

Another example is provided by the 2000 case of eBay vs. Bidder's Edge. ${ }^{5}$ Here, eBay, an online auction site, successfully sued Bidder's Edge, a firm which collected together prices from different auction site for consumers to compare, for cyber-trespass, ostensibly on the grounds that Bidder's Edge spidering activities caused excessive load on their servers. However, as various commentators pointed out the ability to exclude a firm such as Bidder's Edge could also have serious anti-competitive effects ${ }^{6}$. EBay is a classic example of a platform in a two-sided market with sellers taking the role of 'software' or 'service' and buyers that of consumers. If a third-party were easily able to transfer (port) sellers from one auction platform to another then eBay's market power would be greatly diminished. A firm such as Bidder's Edge would greatly facilitate such 'porting' by ensuring that a given seller (and their associated 'reputation') would be visible to consumers no matter what auction platform they were on. By preventing Bidder's Edge (and any other similar firm) from being able to extract data from the eBay site without permission eBay obtained very substantial control of porting from its platform.

A final example comes from the ongoing debate in Europe around interoperability of TPMs/DRMs (Technological Protection Measures/Digital Rights Management) systems, particularly in relation to the dominant position of Apple's iPod and iTunes products both of which use Apple's proprietary 'FairPlay' DRM. Here the platform is the digital music player and the 'software' is the music. Apple operates on both sides of the market with the iPod or iTunes software on the platform side and the iTunes Music Store (ITMS) on the 'software' (music) side. If DRM were interoperable then one could play a song from any given digital music store on any given digital music player. However with no interoperability if someone buys all their songs from the iTunes Music Store (currently with

\footnotetext{
${ }^{4}$ This also explains why Microsoft only 'integrates/ties' certain applications and is happy for most software to be produced by third-party vendors. The need to tie only arises when that application or service will itself be the site of significant third-party development. This is clearly the case with web-browsers, as Bill Gates presciently saw in his 'Internet Tidal Wave' memorandum: "A new competitor 'born' on the Internet is Netscape [Netscape was launched 15th Dec 1994]. Their browser is dominant with $70 \%$ usage share, allowing them to determine what network extensions will catch on. They are pursuing a multi-platform strategy where they move the key API into the client to commoditize the underlying operating system ..." (emphasis added).

${ }^{5}$ EBAY, Inc vs. BIDDER'S EDGE Inc, http://pub.bna.com/lw/21200.htm.

${ }^{6} \mathrm{See}$, for example, the amicus curiae brief filed by a collection of 28 law professors available online at http://www.gseis.ucla.edu/iclp/ebay-ml.
} 
$70-80 \%$ of the digital downloads market) then they can only play them on an iPod (and if they change music player they may lose all their purchased music). Thus proprietary DRM makes it substantially harder for consumers to switch platforms (i.e. digital music players). By maintaining a closed, proprietary, DRM system and integrating backwards into the 'software' (music) market (analogously to the previous Microsoft examples) Apple are able to effectively control porting and thereby increase their market power in the platform (music player) market. ${ }^{7}$

The paper builds upon several strands in the existing literature. First, there is existing work on 'converters' in network markets (converters being devices that allow a user on one network to gain access to a separate network). For example, Farrell and Saloner (1992) examine the provision and purchase of imperfect converters in a network effects model, as well as the incentive for a dominant firm to make conversion costly. ${ }^{8}$ As porting can be seen as the analogous activity in a two-sided market with 'indirect network effects' to converters in the original 'one-sided' models our work can be seen as extending this existing work to the more complex two-sided case.

The second strand is the literature on indirect network effects and two-sided markets. Early work by Church and Gandal (1992) (extended by Church, Gandal, and Krause (2003)) analyzed the case where consumers cared about the variety of complementary goods available for a particular platform or network. They showed that with fixed costs in production this led to 'indirect network effects', that is a positive relation between the utility of a consumer from a given platform and the number of other consumers joining that platform. This work has recently been extended and generalized under the heading of two-sided markets, see, for example, Armstrong (2005); Rochet and Tirole (2003, 2005). The focus of much of this literature has been on the charging decisions of the platform owner - in particular, the form of fees and what determines the level of fees, and subsidies, on the two sides (the 'software' side and the consumer side). By contrast, in this paper we are interested in something rather different: what happens if one platform owner can influence the availability of 'software' on the other platform by controlling porting.

\footnotetext{
${ }^{7}$ It is important to note for this analysis that it is well-known that Apple make their profits on the hardware (the iPod) and make very little from the iTunes Music Store.

${ }^{8}$ See also Choi (1997) for another converter model, albeit a dynamic one related to the transition from an old to a new technology.
} 
Seen in this light, the closest work to ours in the existing two-sided literature are the papers of Armstrong and Wright (2005) and Choi (2006). Armstrong and Wright (2005) provides a general examination of two-sided markets with multi-homing. In particular, they consider the use of exclusive contracts by a platform owner as means to force singlehoming on the seller side. However, due to the complexity of the analysis in the full two-sided the case the authors fall back to analyzing the case of pure network effects. ${ }^{9}$ Our model differs from this in several ways. First, rather than exclusive contracts we have a general 'porting cost' variable which influences the ability of 'software' produced for one platform to move to the other. Second, we allow for ex-ante asymmetry in platform's market share and general forms for both heterogeneity and indirect network effects. However, like Armstrong and Wright (2005), the fully general case is too complex for ready analysis and so the price we pay to keep the model tractable is some degree of restriction on platform's pricing decisions as well as confining ourselves to the case where a single proprietary (and dominant) platform faces a competitive one.

Choi (2006) presents a rather different model, which is primarily animated by the Media Player case, focusing on the combination of tying with multi-homing on the buyer (consumer) side. Here, tying is about the ability for a firm with a monopoly in some underlying market to use tying to monopolize a related two-sided platform (for example Microsoft using its operating system monopoly to control the media players). With multi-homing on the buyer side Choi finds that the welfare effects of tying are ambiguous with tying in some cases being welfare improving. Our concerns are rather different. First, we have 'porting' (multi-homing) on the 'software' (seller) side, not the consumer (buyer) side, of the market. Second, and more importantly, the 'tying' in our model is between the platform and its associated 'software', not between some outside product and the platform.

Finally, our paper obviously has commonalities with the literature on tying and vertical foreclosure (see e.g. Whinston (1990); Bernheim and Whinston (1998)). Due to the prominence of the tying issue in the Microsoft case there has been a flurry of papers on tying models. Perhaps the closest, at least in spirit, to the model presented here is that of Gilbert and Riordan (2007) and Carlton and Waldman (2002).

\footnotetext{
${ }^{9}$ The focus is on the case of symmetric platforms which may be problematic when analyzing tying, as the authors state (p. 22): 'Given the underlying symmetry of firms in our model, it is not obvious that exclusive contracts are advantageous to the platforms in equilibrium.'
} 
Gilbert and Riordan (2007) investigate what they term 'technological' tying by a monopolist. Increasing porting cost in our model could be seen as analogous to the 'technological' tying in their model (whereby the quality of a complementor can be reduced by the monopolist). That said, technological tying is similar to traditional tying in that it is motivated by a desire to sell the complementary good (or the bundle), whereas for the case of porting examined here that is so: the monopolist simply wishes to inhibit complementors from porting to another platform in order to reduce competition with its own platform. Integration, if it happens at all, may occur not because it is profitable in itself - it may even be loss-making - but only because it reduces the degree of platform competition.

Carlton and Waldman (2002), investigate the motivations for a monopolist to tie downstream (in the complementary market) in order to shore up its monopoly in the upstream market. In their model downstream entry in period one, by increasing the available rents upstream, can result in entry upstream in period two. By tying downstream the upstream monopolist may eliminate this possibility thereby preserving its monopoly. The key feature here is that an upstream producer may extract some of the rents of a more efficient downstream producer and therefore may be motivated to enter when the downstream product is not tied. While our analysis shares the important similarity that tying (or manipulating porting cost) is not about the rents in downstream markets but about preserving the upstream monopoly it differs in important ways.

First, our focus is on porting costs not explicit tying. This in turn reflects the fact that a platform's major feature is its very large number of complementors. In such cases it is almost always infeasible for a platform to explicitly tie its associated 'services'. Second, though in both models the monopolist's intervention operates to harm competitors (actual or potential) the mechanism is rather different. In Carlton and Waldman (2002) it is the potential for an improved complementary downstream good to generate rents for upstream firms that motivates tying. Here, the monopolist's motivation for controlling porting (and to explicitly tie in some cases) is related not to the quality of the complementary good but the ease with which it can affect switching (porting) to another platform - thereby 
weakening the monopoly basis in the indirect network effects arising from the platform (two-sided) market structure. ${ }^{10}$

\section{The Model}

The basic framework is that used in the two-sided markets literature (see e.g. Armstrong (2005)). There are two platforms/networks: $X=A, B$ and a mass of consumers (buyers) modelled by the interval $[0,1]$ with the index, $t \in[0,1]$, used to label them. The measure of consumers on platform $X$ is denoted by $n_{X}$. Each platform has an associated set of 'software/services' ('sellers') and the amount of 'software' available on platform X is $s_{X}$.

Consumers derive utility from using software and must purchase access to a platform to be able to use the associated software. ${ }^{11}$ Consumers are heterogeneous in their preferences for a given platform. ${ }^{12}$ If a consumer has already purchased 'software' from one platform she gains no extra utility from purchasing from a second platform so a consumer will purchase from at most one platform (there is no multi-homing on the buyer side). We also make the standard assumption that all consumers join one or other platform.

Formally, consumers have the following utility function:

$$
u_{X}\left(t, p_{X}, s_{X}, p_{X}^{s}\right)=\phi-p_{X}-h_{X}(t)+u_{X}^{s}\left(s_{X}, p_{X}^{s}\right)
$$

Where

- $\phi$ is a positive constant introduced so that reservation utility can be normalized to 0 (alternatively one could remove $\phi$ from utility function and set reservation utility to $-\phi)$

- $p_{X}$ is the price of hardware on platform $\mathrm{X}$

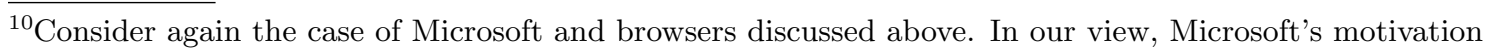
in creating (and tying) Internet Explorer was not that that Netscape Navigator would lead to increased rents for other operating systems producers and hence to entry of competitors, but the fact that Netscape would massively reduce porting costs to alternative platforms of complementary software and content as these types of products moved increasingly online (remember Netscape Navigator was itself cross-platform and hence all online sites that 'ran' on it would implicitly become be themselves cross-platform).

${ }^{11}$ There are no 'direct' network effects, that is consumers' utility from a given platform is affected directly only by the platform itself and the amount of software available on it and not by the number of other consumers using that platform. It would not be difficult to incorporate direct effects into our model but as that is not the focus of our analysis here we have chosen to omit them for the sake of simplicity.

${ }^{12}$ This could be taken as encapsulating general differences in the type of software available on the two platforms.
} 
- $h_{X}(t)$ models consumer heterogeneity. It is assumed that heterogeneity is symmetric across platforms that is, $h_{B}(1-t)=h_{A}(t)$. This allows one to write $h_{A}(t)=h(t)=h_{B}(1-t)$. We shall assume the standard 'orderability' of consumers by heterogeneity, i.e. $h^{\prime}(t)>0$. Thus we have a standard linear city model with platform $\mathrm{A}$ at 0 and platform $\mathrm{B}$ at 1 and consumers preferring, all other things being equal, a closer platform.

- $u_{X}^{s}$ is utility from software purchases with $s_{X}$ the amount of software available on platform $\mathrm{X}$ and $p_{X}^{s}$ the price (or vector of prices) of software. This is discussed further below.

Platform A is controlled by a single firm, the monopolist (M). Platform B is provided competitively. Platform fixed costs are assumed to be sunk and therefore may be taken without loss of generality to be zero. Marginal costs of access per consumer, $c$, are constant and the same for each platform. Since platform B is perfectly competitive the access price equals marginal cost: $p_{B}=c$. Since the marginal cost is common across the two platforms we may, without loss of generality, set $c=0$.

2.1. Software Production and Porting. The software that is produced may be created by two methods. Either it can be created directly for platform $\mathrm{X}$ at fixed cost $f_{X}^{d}$ or it can be ported from the other platform at fixed cost $f_{X}^{p}$ (note that this only relates to the fixed cost, the marginal cost is the same whether the software is ported or created directly). In what follows the main focus will be on the cost of porting from the monopolist's platform (A) and so we will drop the subscript and define $f^{p}=f_{A}^{p}$.

In our model we will suppose that a monopolist may increase the cost of porting from its platform to a competitor's - though at the cost of some expenditure on its own part. ${ }^{13}$ Formally, if $e$ is expenditure then $f^{p}=f^{p}(e)$. It will be convenient in what follows to have the porting cost, $f^{p}$, being the choice variable rather than expenditure, $e$. This simply involves using the inverse function (the expenditure to prevent porting), $e=e\left(f^{p}\right)$. Efforts to prevent porting display diminishing returns so $e^{\prime}\left(f^{p}\right)>0, e^{\prime \prime}\left(f^{p}\right)>0$.

\footnotetext{
$\overline{13}$ For motivation the reader is directed to some of the examples set out in the introduction with perhaps the most relevant one here being the behaviour of Microsoft. Microsoft has incurred significant expenditure on several products, e.g. its Java Virtual Machine, Internet Explorer, the .NET framework, and Windows Media Player, where it would appear that a substantial motivation for the products development was the desire to make it harder to port software and services from its own system to competitors (in each case the product increased Microsoft's control of key APIs and formats).
} 
Thus the fixed cost of software production on a platform, $f_{X}$, will be either: $f_{X}^{d}$ if all software is produced directly (none is ported); a mixture of $f_{X}^{d}$ and $f_{X}^{p}$ if some software is ported and some produced directly; or $f_{X}^{p}$ if all software is ported.

\subsection{Sequence of Actions.}

(1) The monopolist, $\mathrm{M}$, chooses values for control variables: $p_{A}, f^{p}$.

(2) Next in parallel, consumers and software producers make their choices. Consumers, based on their (common) expectations of the level of software provision and prices, decide from which platform to purchase. At the same time, software firms, based on their expectations of platform size (i.e. the number of consumers on each platform), decide whether to engage in direct software production for (their) platform. Then, given this amount of direct production, (other) producers ${ }^{14}$ decide whether to engage in porting of this existing, directly produced, software.

(3) Payoffs are realized. In particular, M's profits, $\Pi=p_{A} \cdot n_{A}\left(p_{A}, f^{p}\right)-e\left(f^{p}\right)$, are determined.

Remark 1. In equilibrium the resulting platform sizes must be consistent with rational expectations. That is: actual and expected platform sizes are equal and actual and expected software levels are equal. In this case the order in which software firms and consumers move does not affect the equilbrium outcome of the model. Thus we could as easily have software firms taking their decisions after consumers or vice-versa.

For those requiring justification on this point consider first the sequential setup. Suppose consumers move first. Then given consumer platform choices software provision will be uniquely determined by software firms profit-maximization problems. Conversely suppose software providers move first. Then, given software provision, consumers' choice of platform will be uniquely determined by their utility maximization condition. Thus in a sequential setup the actions of the group that moved second would be entirely determined by the those that moved first. One could then substitute this back and would end up with a classic 'indirect network-effects' coordination game for the group that moved first.

\footnotetext{
${ }^{14}$ Here it will not matter whether the firms that engage both in direct production and porting are the same or different since direct production confers no special ability in porting and, with free entry, all producers earn zero profits in equilibrium.
} 
In the case of simultaneous moves, rational-expectations, by eliminating the possibilty of 'mis-match' between beliefs and outcomes, in essence, make the actions of one group dependent on that of the other just as in the sequential move case. For example, suppose consumers have expectations of software provision $\mathrm{E}$ and based on this the platform sizes are $\mathrm{X}$. Then, in equilibrium, a) software firms expectation of platform size E' must equal X - otherwise software firms expectations are incorrect and b) given $\mathrm{X}$ the equilibrium level of software provision must be $\mathrm{E}$ - otherwise consumers expectations were incorrect. Of course, the sequence of actions will alter which group finds itself in the 'driving seat' but this will not alter (at last in this model) the equilibrium outcomes (in terms of platform size and software provision) - which is what matters to the monopolist, and therefore to us. Given this indifference as to which group is accorded the 'driving-seat' we are free to make a choice and in what follows we shall generally accord consumers that position and take softare provision as determined (implicitly) by consumers' actions.

\section{Solving The Model}

We take a general approach in which we assume only that software production on platform X involves (a) some form of fixed costs $\left(f_{X}\right)$ (b) that the amount and price of software on platform $X$ may be expressed solely in terms of these fixed costs, $f_{X}$ and the number of consumers on the platform, $n_{X}$. Taken together these mean that the consumer software utility function has a reduced form of the following kind:

$$
\begin{aligned}
u_{X}^{s}\left(s_{X}, p_{X}^{s}\right) & =u_{X}^{s}\left(s_{X}\left(f_{X}, n_{X}\right), p_{X}^{s}\left(f_{X}, n_{X}\right)\right) \\
& \equiv \nu_{X}\left(f_{X}, n_{X}\right) \text { with } \nu_{f_{X}}<0, \nu_{n_{X}}>0
\end{aligned}
$$

We shall term $\nu_{X}$ the 'indirect network effects' function on platform X. ${ }^{15}$ By proceeding in this manner the results are kept as general as possible. Furthermore, the two basic models of imperfect competition with fixed costs (monopolistic competition and product differentiation) can both be shown to give rise to this reduced form (Appendix B provides an explicit derivation for the case of a standard circular city model of product differentiation).

\footnotetext{
${ }^{15}$ Note that we implicitly assume some symmetry across platforms in that the function $\nu$ is the same for the two platforms.
} 
As presented we now have a standard two-sided model with utility functions:

$$
u_{X}\left(t, p_{X}, f_{X}, n_{X}\right)=\phi-p_{X}-h_{X}(t)+\nu\left(f_{X}, n_{X}\right)
$$

We can solve this in the usual manner to obtain platform sizes as a function of the monopolist's choice variables: $n_{A}=n_{A}\left(p_{A}, f^{p}\right) .{ }^{16}$ The monopolist then solves:

$$
\max _{p_{A}, f^{p}} p_{A} n_{A}\left(p_{A}, f^{p}\right)-e\left(f^{p}\right)
$$

3.1. Solving for the Subgame Equilibrium. We solve first for the equilibrium platform size in the consumer/software subgame (stage 2 onwards, that is after $M$ has set prices and porting cost). We proceed by the usual method based on finding the marginal consumer indifferent between the two platforms.

First, recall that we have assumed that consumers gain no extra utility by purchasing from more than one platform. Thus, we may assume that consumers purchase at most one platform. We also assumed that all consumers do purchase from one or other platform. Thus we have $n_{B}=1-n_{A}$ and we need only consider $n_{A}$ in what follows. For notational convenience suppress auxiliary variables in the consumer utility functions and write $u_{X}\left(t, p_{X}, f_{X}, n_{X}\right)=u_{X}\left(t, n_{X}\right)$.

Define: the conditional utility advantage of platform A over platform B for consumer $t$ when platform size is $n_{A}$ :

$$
\hat{A}\left(t, n_{A}\right)=u_{A}\left(t, n_{A}\right)-u_{B}\left(t, 1-n_{A}\right)
$$

and the utility advantage (function), which gives the utility advantage of platform A over $\mathrm{B}$ if $\mathrm{t}$ is the marginal consumer $\left(\right.$ so $\left.t=n_{A}\right)$ :

$$
A(t)=\hat{A}(t, t)
$$

Using the expression for the utility function we have that:

$$
A(t)=-p_{A}-h_{A}(t)+h_{B}(t)+\nu\left(f_{A}, t\right)-\nu\left(f_{B}, 1-t\right)
$$

\footnotetext{
${ }^{16} n_{A}$ will also depend on other variables such as the direct cost of software production but these are exogenous variables not under the control of any player.
} 
Lemma 2. The equilibria of the subgame from stage 2 onwards (after $M$ sets price and porting costs) are given by $E=E_{0} \cup E_{-0}$ where $E_{0}$ is the set of interior equilibrium, $E_{0}=\{t: A(t)=0\}$, and $E_{-0}$ is the set of extremal or 'standardization' equilibrium in which all consumers join one or other platform, $E_{-0}=\{0: A(0)<0\} \cup\{1: A(1)>0\}$. An equilibrium $t_{e} \in E_{0}$ is stable if $A^{\prime}\left(t_{e}\right)<0$. All $t_{e} \in E_{-0}$ are stable.

Proof. See appendix.

Note that the advantage function implicitly depends on all of our exogenous and choice variables: $A(t)=A\left(t, p_{A}, f_{A}, f_{B}\right)$ and therefore so does the set of equilibria $E=E\left(p_{A}, f_{A}, f_{B}\right)$. We make the following assumption about the existence of an equilibrium to this subgame: Assumption: The exogenous variables, in this case the functional forms for the heterogeneity and indirect network effects, are such that, when $p_{A}=0$ and the porting cost at its initial value (that is without any intervention by $\mathrm{M}$ ), there would exist an asymmetric stable interior equilibrium where platform $\mathrm{A}$ is larger than $\mathrm{B}$.

Justification: without a stable equilibrium of the subgame the overall game will clearly have no equilibrium. Thus we must have the existence of at least one stable equilibrium of the subgame. ${ }^{17}$ We require the existence of an interior stable equilibrium to the subgame for two reasons. First, in most real-world scenarios, even those that involve a very dominant platform, we rarely see a platform with $100 \%$ market share. Second, at an extremal equilibria the monopolist actions no longer have a marginal impact (for example, the monopolist may increase or decrease prices without any impact on demand). This renders such equilibria both less interesting and more cumbersome to analyze. Finally, with regard to the asymmetry: in most real world situations one platform is larger than the other. Furthermore, in any situation with antitrust considerations this will necessarily be the case.

3.2. Porting. In this section we shall determine the amount of software produced for each platform of the various possible types (produced directly, ported or produced by a mixture of those methods). In doing so, we will also have determined the 'actual' fixed

\footnotetext{
${ }^{17}$ This part need not be an assumption since under mild conditions, such as symmetry of the indirect network effects and heterogeneity function, one can show there exists at least one stable equilibrium to the subgame. However it is clearly not possible to ensure the existence of a stable interior equilibrium in general - consider the standard symmetric case with linear heterogeneity and network effects: he only interior equilibrium is at 0.5 and with 'strong' network effects this equilibrium must be unstable.
} 
cost of software production for each platform $f_{A}, f_{B}$ in terms of the fixed cost of directly producing software for that platform and the (common) porting cost $\left(f_{X}^{d}, f^{p}\right)$. To simplify the statement of results it will be useful to make a technical assumption to exclude one particular measure zero configuration of (expected) platform sizes and direct software production costs:

Technical Assumption: $f_{A}^{d} n_{B} \neq n_{A} f_{B}^{d}$.

Lemma 3 (Porting Lemma). In equilibrium only one platform has software produced directly for it. All the software on the other platform derives from porting. Let $X$ denote the platform for which software is produced directly and denote the other by $X$ '. Then $f_{X}=f_{X}^{d}$ and the amount of software on $X^{\prime}$ will be equal to the smaller of:

(1) The amount of software on $X$ (in the case where all software is ported)

(2) The level of software production is determined by the porting cost, i.e. the level of software production is that which would be produced with $f_{X^{\prime}}=f^{p}$.

If the second case obtains, i.e. not all possible software is ported (so the level of porting cost matters), the porting constraint will be said to bind and we have $f_{X^{\prime}}=f^{p}$.

Proof. See appendix.

Remark 4 . The result that, for any given platform, all software is either produced directly or ported may seem a little implausible. After all, in reality, we usually see software produced directly for all platforms. It is also usual for there to be substantial porting, with the same piece of software available on multiple platforms (multi-homing on the software side). ${ }^{18}$ However, all that is necessary for the results in this paper is that the marginal piece of software on the platform competing with the monopolist is ported - in which case altering the costs of porting change the amount of software on that platform. Thus, while the model as given may seem to be overly restrictive in its implications the necessary result, that is that the porting constraint binds, will still hold in the more general case.

We now make one further assumption:

\footnotetext{
${ }^{18}$ Extending the model to have direct production on both platforms and intermediate levels of of porting could most easily be done by allowing heterogeneity in both direct production and porting costs of 'software'.
} 
Assumption: In the case of asymmetry, it is the platform with larger (expected) size for which software is produced directly.

Justification: we have just shown that it will always be the case (in this model) that software on one platform has all software produced directly and one has all software ported. Since the amount of software on the 'porting' platform must always be less than or equal to that on the 'direct-production' platform it is natural to assume that it is the platform with larger (expected) size for which software is produced directly. ${ }^{19}$

Combining these assumptions with the results of the previous section we may set $f_{A}=$ $f_{A}^{d}$ and $f_{B}=f^{p}$ (though we will need to check that the porting constraint does not bind).

3.3. Solving for Overall Equilibrium. Finally it is necessary to demonstrate the existence of an equilibrium in the overall game: that is a solution to the monopolist's profit maximization problem taking account of how the monopolist's choices impact on the actions that will be taken by other agents (consumers and software producers). This response of other agents to M's choices has already been derived in the form of the subgame 'network' equilibrium derived above. We note that these results may not be easy to grasp when presented as generally as they are here. The following section examines a specific case graphically and the reader may find it profitable to peruse that example first before returning to the more abstract approach used here.

Lemma 5. Having picked a stable interior equilibrium platform size $t_{e}^{0} \in E_{0}\left(p_{A}^{0}, f_{A}, f_{B}\right)$ we have associated to it a well-defined, continuous and differentiable 'platform size function', $t_{e}\left(p_{A}, f_{A}, f_{B}\right)$, defined in a neighbourhood of $t_{e}^{0}$. In particular, restricting to changes in $p_{A}$ we have a demand function for the Monopolist:

$$
q\left(p_{A}\right)=t_{e}\left(p_{A}\right)=A^{-1}\left(p_{A}\right)
$$

Differentiating we have:

(1) Downward sloping demand schedule: $\frac{d q}{d p_{A}}=\frac{-1}{A^{\prime}\left(t_{e}\left(p_{A}\right)\right) t_{e}^{\prime}\left(p_{A}\right)}<0$

(2) $\frac{d t_{e}}{d f_{A}}<0$

(3) $\frac{d t_{e}}{d f_{B}}>0$

\footnotetext{
${ }^{19}$ In fact if platforms displayed symmetry, i.e. direct production costs are equal and heterogeneity functions on the two platforms are the same, this is a result rather than an assumption.
} 
Finally, though demand may be discontinuous at some point, there exists locally, that is within the region where demand is continuous, a unique profit maximizing price.

Proof. See appendix.

Combined with the results of the previous section the monopolist's profit maximization problem becomes:

$$
\max _{p_{A}, f^{p}} p_{A} \cdot t_{e}\left(p_{A}, f^{p}\right)-e\left(f^{p}\right)
$$

We make one final additional technical assumption which allows us to rule out the possibility of discontinuities in M's profit function as a result of changes in porting cost: Assumption: Pick such an asymmetric stable interior equilibrium $t_{e}^{0}$ and consider the associated equilibrium function $t_{e}\left(p_{A}, f^{p}\right)$. Then that function exists and is continuous for all values of $f^{p}$ up to $f_{B}^{d}$ (which is the maximal value that $f^{p}$ would ever be set to by $\mathrm{M}$ ).

Corollary 6. There exists an equilibrium of the overall game, that is a price and porting cost and an associated equilibrium level of demand $t^{e}\left(p_{A}, f^{p}\right)$ which maximize the monopolist's profits.

Proof. See appendix.

3.4. Example I: Equilibrium and Demand. The situation we shall consider is one in which the two platforms are a priori equivalent, that is the fixed costs of software production on the two platforms are equal and heterogeneity is symmetric $\left(h_{B}(1-t)=\right.$ $\left.h_{A}(t)\right) .{ }^{20}$ For the 'network effects' function we use the reduced form derived from a circular city model (see appendix), that is $\nu(f, t)=C-\sqrt{\frac{f}{t}}$. This form differs substantially from the classic, linear, network effects functions found usually in the literature. There are several reasons to choose this more complex form as opposed to a simpler linear one. First, this function is founded on an explicit derivation from a particular model of competition in the software market. Second, the linear form, at least when coupled with a linear heterogeneity function (as is usually the case), severely limits the form and set of possible equilibria - most obviously there is only one interior equilibrium configuration and this is necessarily symmetric (if network effects and heterogeneity are symmetric, i.e. a priori the

\footnotetext{
${ }^{20}$ It has already been assumed above that the network effects are symmetric, that is $\nu_{A}=\nu_{B}$.
} 
platforms are equivalent). Third, and most importantly, as we discuss in detail below, the form of the network effects function is a key determinant of comparative statics for welfare. In this regard, the use of the linear form is not 'innocent' and has strong implications for the results. While this is obviously true of any other form chosen, including the one here, examining a slightly more complex, and less standard case, forces us to think more carefully about the implications of choosing one particular functional form over another.

Coming to the heterogeneity function it is set to take the form $h_{A}(t)=10 t^{10}$. This corresponds to a situation where there is a large middle ground of consumers who are fairly indifferent between the two platforms $(\mathrm{h}(\mathrm{t})$ is small until t is close to 1$)$ but two 'extreme' groups at either end who have strong preferences for their nearest platform. The high power $\left(t^{10}\right)$ was determined by the need to ensure the existence of a stable asymmetric equilibrium and itself reflects the sharp concavity of the network effects function. Fixed costs are set as follows $f_{B}=f_{A}=1.5$. These values are chosen so as to generate a stable asymmetric equilibrium as shown in Figure 1. Note that in its general shape (i.e. number of equilibria, location of maxima/minima) this graph is the simplest possible that gives rise to a stable asymmetric equilibrium. ${ }^{21}$

3.4.1. Discontinuity of demand: Since price enters $A(t)$ linearly the diagram above also implicitly defines the demand function in the neighbourhood of an equilibrium (an increase in the $p_{A}$ shifts the $A(t)$ curve down by that amount). A maximum of $A(t)$ therefore corresponds to a point at which demand is discontinuous (as price rises above the maximum value demand jumps down as the market tips to the neighbourhood of next lowest stable equilibrium).

An illustration of this is provided in Figure 2, which plots the demand function derived from Figure 1 in the neighbourhood of the stable equilibrium at 0.84. Here demand is discontinuous at a price just below 0.5 (i.e. at the left edge of the diagram - the discontinuity itself is not shown as it distorts the scale). At the discontinuity demand will suddenly jump down to approximately 0.14 which is the next place the line $\mathrm{y}=0.5$

\footnotetext{
${ }^{21}$ To have an interior stable equilibrium $A(t)$ must intersect the line $y=0$ from above. If heterogeneity is symmetric, $h_{A}(t)=h_{B}(1-t)=h(t)$ then when fixed costs are equal and prices are zero, $A(t)$ must be anti-symmetric about 0.5 , i.e. $A(t)=-A(1-t)$. This implies $A(0.5)=0$ so 0.5 is an equilibrium. Thus with symmetry in the network function and assuming that standardization equilibria exist (i.e. 0 and 1 are equilibrium) the fewest crossings (i.e. interior equilibria) that lead to the existence of a stable asymmetric equilibrium is five and we must have a situation similar to that shown.
} 


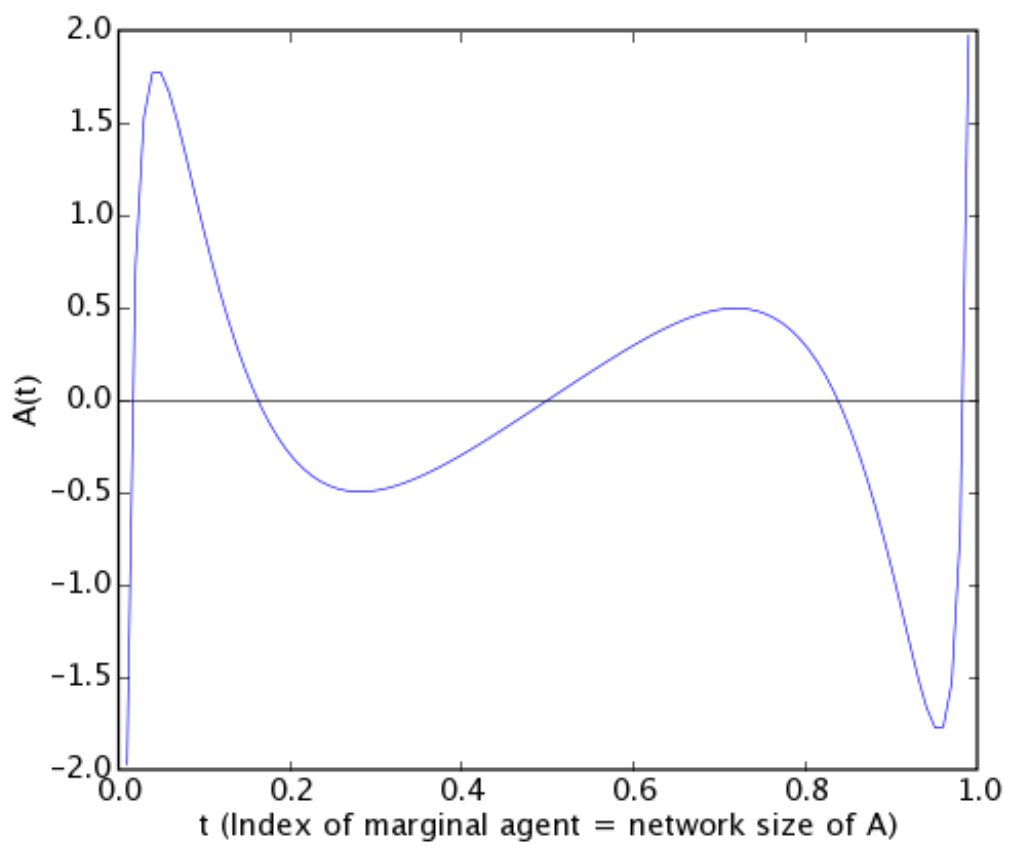

Figure 1. The utility advantage function, $A(t)$ in the symmetric case when the access prices for the two platforms are the same (so $p_{A}=0$ ) and $f_{A}=f_{B}=1.5$. There are stable equilibria at 0 and 1 (the 'standardization' equilibria) and 0.16 and 0.84 (asymmetric stable equilibria). There are unstable equilibria at 0.5 and 0.02 and 0.98 .

would intersect $A(t)$ (see Figure 1). Note how this diagram is just the relevant portion of Figure 1 between 0.73 and 0.84 'blown up'.

In all cases where there is symmetry and a stable asymmetric equilibrium $A(t)$ must have a bounded maximum just like it does in Figure 1. A bounded maximum in turn implies a discontinuity in the demand function of the monopolist. Thus, in all such cases, a monopolist will face a discontinuous demand function. This discontinuity in demand does not exist in the traditional linear network effects models and it functions here to place a sharp upper bound on the price the monopolist can charge without a sudden jump downwards in market share.

3.4.2. Other Comparative Statics: We can evaluate the effect of changing production and porting costs by considering how it shifts $A(t)$. In particular, increasing fixed costs of software production for $\mathrm{A} f_{A}$ will shift $A(t)$ down and increasing $f_{B}$ will have the opposite effect (note that $f_{B}$ is equal to the porting costs, $f^{p}$ if the porting constraint does not 


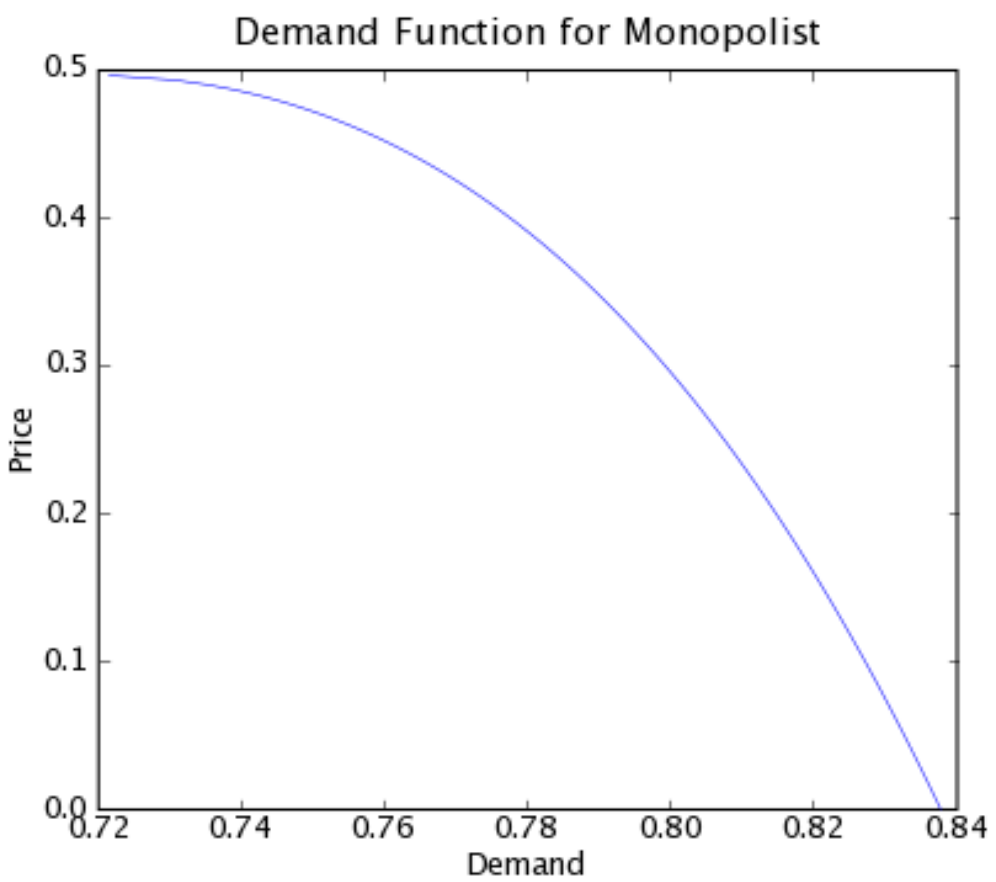

Figure 2. The Demand function for the monopolist in the neighbourhood of the stable equilibrium at 0.84 . Demand is discontinuous at a price just below 0.5 (i.e. at the left edge of the diagram - the discontinuity itself is not shown as it distorts the scale).

bind). Note that unlike price, fixed costs do not enter linearly so they will also change the shape of $A(t)$ and not just its level.

\section{WELFARE}

Having established the various properties of equilibrium in this section we come to the central questions of this paper: how does the monopolist's control of prices and the cost of porting affect consumer and social welfare? Giving equal weight to monopoly profits and consumer welfare we have that total welfare, $W=\Pi_{A}+W^{C}$ where $W^{C}$ is consumer welfare and $\Pi_{A}$ are the monopolist's profits. ${ }^{22}$

Lemma 7. The marginal change in consumer welfare as a function of platform A's size (t) is:

$$
\frac{d W^{C}}{d t}=A(t)+\mu(t)
$$

\footnotetext{
${ }^{22}$ We have assumed overall profits are zero in the software industry as a result of free entry.
} 
where $A(t)$ is the utility advantage of $A$ over $B$ defined previously and

$$
\mu(t) \equiv t \nu_{A}^{\prime}(t)-(1-t) \nu_{B}^{\prime}(1-t)
$$

At an interior equilibrium $t_{e} \in(0,1), A\left(t_{e}\right)=0$, and this reduces to:

$$
\frac{d W^{C}}{d t}=\mu\left(t_{e}\right)
$$

Proof. See appendix.

A first point to emphasize is that this result (and Lemma 8 below) are entirely general and will hold in any model in which consumer utility incorporates a 'network effects' function (whether arising directly, or, indirectly as a reduced form derived from a more complex model). That is, there is nothing that depends on the specifics of the porting framework as presented in this paper. In particular, these results would apply both traditional direct network effects models of communication networks and some of the more recent models arising from a two-sided market structure.

The second point to make is that this result is telling us that, when at an interior equilibrium $\left(x=t_{e}\right)$, the marginal change in consumer welfare with respect to platform size is a function of 'network effects' alone (encapsulated in $\mu$ ). The two basic possibilities, namely that consumer welfare is increasing $\left(\mu\left(t_{e}\right)>0\right)$ or that it is decreasing $\left(\mu\left(t_{e}\right)<\right.$ 0 ) with the size of platform A have a simple interpretation. In the first case we have a situation in which more standardization (that is more consumers on platform A) is preferable. In the second case we have a situation in which more symmetrical platform shares are preferable. ${ }^{23}$

What determines the sign of $\mu\left(t_{e}\right)$ - that is whether standardization or symmetry is preferable? Answer: the degree of curvature of the indirect network effects function, $\nu$, which in more economic terminology could be put as: how sharp are the diminishing returns to network effects in platform size, that is, how fast does the benefit of a new user fall as the number of users on the platform increases - crudely how much (less) does an existing user of a platform benefit as the millionth person joins a platform compared to when the tenth person joins?).

${ }^{23}$ There is, also the third possibility that the change in consumer welfare is zero but this is obviously a very special case. 
Interestingly it turns out that the dividing line between the two cases is where network effects take the form of the natural logarithm: $\nu(x)=C+\ln (x)$. When marginal network effects fall with platform size more gradually than this then $\mu>0$ and standardization is preferable. When marginal network effects fall more strongly than this then $\mu<0$ and symmetry is preferable. The classic form studied in the literature is of course where $\nu$ is linear in which case marginal network effects do not fall at all with platform size and so $\mu>0$ and standardization is preferable. Conversely, the circular city model of indirect network effects studied in the appendix gives rise to the case where $\nu(x) \propto-1 / \sqrt{x}$. In this case marginal network effects fall more sharply than for the logarithm and so $\mu<0 .{ }^{24}$

To summarize, network effects which display weakly diminishing returns imply that standardization (everyone on one platform) will be preferable while if network effects show strongly diminishing returns, a more symmetric platform configuration is preferable. We now proceed to work formally through the consequences of this basic result in relation to the model at hand in the Lemmas below, with the main results summarized in Table 1.

Lemma 8. At a subgame equilibrium, $t_{e}$, the effect on consumer welfare of an increase in the price charged by the monopolist is negative if $\mu\left(t_{e}\right) \geq 0$ and is ambiguous otherwise depending on the relative magnitudes of the monopoly pricing effect (-ve) and the network externality (+ve). Furthermore, at an equilibrium of the overall game (i.e. where the monopolist is profit-maximizing) the change in total welfare equals that in consumer welfare and therefore has the same properties.

Proof. See appendix.

Monopoly pricing does not result in traditional deadweight losses since total demand is fixed and does not change (consumers who leave one platform join the other). ${ }^{25}$ However,

\footnotetext{
${ }^{24}$ The literature on the functional form of the network effects is rather sparse. Both Swann (2002) and Odlyzko and Tilly (2005) provide a thoughtful critique of existing assumptions regarding the form of the network effects function such as that embodied in Metcalfe's law (Metcalfe's law corresponds to the linear case $\nu(x)=x$ ). Swann (2002) focuses on the case of a simple telephone network. He establishes (the fairly restrictive) conditions under which network effects are linear and suggests that when these conditions are not satisfied, network effects will be S-shaped. Odlyzko and Tilly (2005), also consider the linear form unlikely. As a replacement they propose using the logarithmic form, $\nu(x)=\ln (x)$. As we have just shown this is a very special case in which at an equilibrium we have $\mu=0$ and therefore consumer welfare is neither increasing or decreasing in platform size. Clearly, one would like to determine the exact form of the (indirect) platform effects function empirically. However, at least to our knowledge, there are no empirical papers which deal with this issue.

${ }^{25}$ This explains why at full equilibrium marginal consumer welfare and total welfare are equal.
} 
it does shift consumers away from the monopolist's platform (an effect exacerbated by the feedback from the indirect network effects). In markets with 'externalities' such as these this will have consequences for welfare.

The effect of an increase in the monopolist's price depends on two distinct factors. The first factor is the simple one that higher prices reduce consumer welfare because consumers pay more. The second factor is more subtle. An increase in M's price moves consumers off A onto B. This effect may either be negative or positive depending, respectively, on whether a more standardization-type or a more symmetric platform configuration is better for welfare. As shown in Lemma 7 this second condition is equivalent to asking whether $\mu\left(t_{e}\right)$ is positive (standardization-type better) or negative (symmetric better). Thus, if $\mu\left(t_{e}\right)$ is positive, an increase in the monopoly price by reducing the size of platform $\mathrm{A}$ acts to reduce welfare. Conversely when more symmetric platform sizes are preferred then an increase in the monopoly price by reducing the size of platform A actually acts to increase welfare.

If we combine the two factors then we only get an unambiguous prediction (increase in prices reduces welfare) in the first case, that is when a more standardization-type platform configuration is preferable. In the second case, where a more symmetric platform configuration is preferable, the effect will be ambiguous and welfare could actually rise due to an increase in the monopolist's prices.

\begin{tabular}{|c|c|c|}
\hline & Low Curvature & High Curvature \\
\hline Direct Impact of Higher Price & - & - \\
\hline Indirect Impact of Higher Price & - & + \\
\hline Overall Impact of Higher Price & - & O \\
\hline \hline Direct Impact of Higher Porting Cost & - & - \\
\hline Indirect Impact of Higher Porting Cost & + & - \\
\hline Overall Impact of Higher Porting Cost & O & - \\
\hline
\end{tabular}

TABlE 1. Welfare Impact of Changes in Price and Porting Cost. This table summarizes the results of Lemmas 8 and 9. Curvature refers to the curvature of the network effects function in the neighbourhood of an equilibrium (note that at an equilibrium consumer and social welfare are equal). ' $\mathrm{O}$ ' indicates the effect is ambiguous.

Lemma 9. At a subgame equilibrium, $t_{e}$, the effect on consumer welfare of a increase in porting costs is negative if $\mu\left(t_{e}\right)<0$ and is ambiguous otherwise depending on the relative magnitudes of the welfare loss from a direct reduction in software provision on platform $B$ 
and the welfare gain from an increase in A's market share. Furthermore, at an equilibrium of the overall game (i.e. where the monopolist is profit-maximizing) the marginal effect on total welfare equals the marginal effect on consumer welfare.

Proof. See appendix.

Again we have two distinct effects of higher porting costs. The first, and the direct one, is that higher porting costs result in a reduction in availability of software for those on platform B (and probably higher prices too - though this may depend on the specifics of the model for software provision). This unambiguously reduces welfare because higher porting costs mean less software for B users (holding platform B's share constant).

The second effect arises from the fact that, as a result of the change in software availability on B, some consumers move from platform B to platform A. This change is an exactly similar one to that already analyzed above when discussing the effect of a price rise (except here an increase porting cost increases the size of platform A while an increase in price reduces the size of platform A). In particular the effect will be negative if, and only if, $\mu\left(t_{e}\right)$ is negative (more symmetric platform configuration preferred). In this case, both effects operate in the same direction and an increase in porting cost is unambiguously harmful to consumer welfare. On the other hand if a more standardization-type platform is preferable $\left(\mu\left(t_{e}\right)>0\right)$ then this effect is positive and the overall impact on welfare will depend on the relative magnitude of the two effects. In this second ambiguous case, we can explore the 'second order' comparative statics in more detail, and this is done in the next Lemma.

Lemma 10. At a subgame equilibrium, $t_{e}$, if $\mu\left(t^{e}\right) \geq 0$ so that the effect of porting costs on consumer welfare is ambiguous, then it is more likely that the effect is negative:

- The larger is platform B's market share (more consumers to suffer from the reduction on software provision on B)

- The larger is the direct impact of higher porting costs on the provision of software for $B$ (greater reduction on software provision on $B$ ).

- The smaller is the impact of changes in porting cost on A's market share. ${ }^{26}$

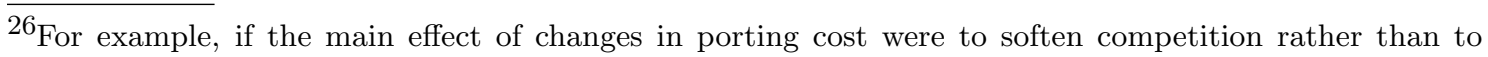
directly increase A's market share. That is, in terms of A's demand curve, increasing porting costs steepened the demand curve or shifted it up but did not shift it out.
} 
- The smaller is the increase in consumer welfare of an increase in A's market share.

Proof. See appendix.

\section{EXAmple II: Welfare}

We now return to our previous specific example, this time in order to illustrate the welfare analysis. Using it, among other results, we demonstrate that it is possible for the welfare costs (consumer or societal) of the control of porting to be significantly greater than the costs of monopoly pricing.

We first choose specific functional forms and values for constants. The heterogeneity function is chosen to ensure that there exists an asymmetric stable equilibrium and is the same as that used for figure 2 above: $h(t)=10 t^{10}$.

The direct costs of software production are set to $f_{A}=1.5$ and the initial porting cost is set to two-thirds of that value, so $f^{p}=1.0$. The monopolist's expenditure function is: $e\left(f^{p}\right)=2 \cdot\left(f^{p}-1\right)^{4}$ and the initial value of $f^{p}$ when there are no efforts by the monopolist is set to 1 . The expenditure function displays diminishing returns and while initial efforts to prevent porting are relatively cheap the cost then escalates rapidly.

The exact parameters for the functional form of the expenditure function are chosen so that an interior 'porting cost' solution exists i.e. the value of porting cost obtained is such that $f_{A}>f^{p}$ and expenditure to prevent porting is non-zero and non-infinite. Using these values we can now proceed to solve the monopolist's problem by numerical means and have the following results.

We find the values chosen for the two control variables are 1.419 for porting costs and 0.43 for the access price of platform A. We also calculate the profit-maximizing price M would charge when unable to influence porting costs: 0.079 . Our main interest is in the significance of M's choices for welfare and welfare outcomes. These, along with the values of other significant variables, are presented in Table 1 (NB: since $\phi$ is an arbitrary constant it has been set so that initial welfare values are normalized to zero. This value has no significance since, as already explained, welfare can be changed by a fixed constant $(\phi)$. Thus only the sizes of welfare changes can be meaningfully compared.)

The first line is there to show the baseline case, when the control parameters are at their 'default' values (that is without intervention by the monopolist). In this case, M's 


\begin{tabular}{|c|c|c|c|c|c|l|}
\hline & $\begin{array}{c}\text { Porting } \\
\text { Cost }\end{array}$ & $\begin{array}{c}\text { Price of A } \\
\text { Hardware }\end{array}$ & $\begin{array}{c}\text { A's } \\
\text { market } \\
\text { share }\end{array}$ & $\begin{array}{c}\text { Net } \\
\text { Profits for } \\
\text { M }\end{array}$ & $\begin{array}{c}\text { Consumer } \\
\text { Welfare }\end{array}$ & $\begin{array}{l}\text { Total } \\
\text { Welfare }\end{array}$ \\
\hline $\begin{array}{c}\text { Initial porting } \\
\text { cost, } \\
\text { competitive } \\
\text { prices }\end{array}$ & 1.0 & 0 & 0.758 & 0 & 0.0 & 0.0 \\
\hline $\begin{array}{c}\text { Initial porting } \\
\text { cost, monopoly } \\
\text { price on A }\end{array}$ & 1.0 & 0.079 & 0.704 & 0.056 & -0.046 & 0.010 \\
\hline $\begin{array}{c}\text { Monopolist } \\
\text { chosen porting } \\
\text { cost, monopoly } \\
\text { price on A }\end{array}$ & 1.419 & 0.43 & 0.729 & 0.252 & -0.406 & -0.154 \\
\hline
\end{tabular}

TABle 2. Welfare Results at Various Prices and Porting Costs

market share, with its own price at zero and the fixed costs of porting at 1, is still $75 \%$. Total welfare and consumer welfare are the same - since prices are zero - and has been normalized to zero.

The next line shows the situation if the monopolist can only set prices and is not able to influence porting costs. This helps us benchmark the relative gain to a monopolist of being able to influence porting costs in addition to setting prices. In line with theory the welfare change is slightly positive, reflecting the reduction in the size of Network A.

The final line shows the actual outcome with the porting cost and price at the level chosen by $\mathrm{M}$ to maximize its profits. Porting costs increase by almost a half to 1.42 , nearly reaching the same levels as the cost of direct production (1.5). Prices rise by over five times compared to the situation when porting costs can not be altered demonstrating the large impact of the Monopolist's control of porting. Despite the far higher price, market share for the monopolist rises though it is still lower than in the situation where neither price nor porting cost can be set.

5.1. The Monopolist's Profits. M gains dramatically from the ability to manipulate porting costs, the percentage increase in profits being approximately $400 \%$ over what is obtained when porting costs are fixed. Moreover this is net of the costs incurred to prevent porting, $e\left(f^{p}\right)=0.0616$, which are equal to a fifth of gross profits. The main effect of raising porting costs is not to increase market share but to soften competition between the two platforms and therefore permit a much higher profit-maximizing price to be charged. 
Market share at the monopoly price in the two cases when porting cost is and is not manipulatable are quite close (0.704 vs. 0.729).

5.2. Consumer welfare. The change in consumer welfare from monopoly pricing, $\Delta W_{c}^{M}=$ -0.046. The change resulting from higher pricing and higher porting costs is $\Delta W_{c}^{M f}=$ -0.406 . Thus consumer welfare losses arising from the combination of higher porting costs and higher prices are almost nine times as large as those arising from higher prices alone. ${ }^{27}$

5.3. Total welfare. For total welfare increasing M's price will actually increase welfare: with porting cost at $1, \Delta W^{M}=0.01$. However the welfare change due to the combination of monopoly pricing and higher porting costs is decidedly negative $\Delta W^{M f}=-0.156$. Thus for this case welfare costs go from barely positive to significantly negative.

5.4. Alternative Specifications. This example is of course based on only one set of functional forms and one set of parameter values among many. It is therefore natural ask how specific the estimates presented here are to those particular choices.

In many ways we are rather limited in what we can say: those general results that are obtainable have already been presented in the previous section. As shown there the welfare impact of a change in price and porting cost depend crucially on the rate of diminishing returns of the network effects function. With strongly diminishing returns pricing has an ambiguous impact but porting costs have a negative impact but with weakly diminishing returns we have the converse: a negative price impact and an ambiguous impact of porting cost.

Thus the choice of network effects function to use in a simulation will clearly influence the estimated welfare impact. The example here uses an indirect network effects function which displays strongly diminishing returns - and consistent with the general results we find a weakly positive impact of pricing and a negative impact of porting cost. However if one were to use a network effects function with weakly diminishing returns (for example

\footnotetext{
${ }^{27}$ As already stated, as welfare is only defined up to a constant we can only compare changes in welfare and not levels. Nevertheless, utility is money metric (prices enter linearly) and profits are well-defined so it is possible to convert of welfare changes into monetary terms. As a very simple 'back-of-the-envelope' calculation consider applying this analysis to the Microsoft case. Profits in 2000 (around the time of the antitrust settlement in the US) were approximately $\$ 9.5$ billion and in our model profits equal 0.252 . Thus, in dollar terms the change in consumer welfare from monopoly pricing alone equals approximately $\$ 1.7$ billion $(0.046 / 0.252 \cdot 9.5)$, while the change in consumer welfare with both higher prices and higher porting costs equals $\$ 15.3$ billion $(0.406 / 0.252 \cdot 9.5)$.
} 
linear network effects) this would likely change the results - it would certainly make it more likely that the pricing impact on welfare was more significant than the porting impact. ${ }^{28}$

\section{Conclusion}

In this paper, we introduced 'porting' into a standard, two-sided, indirect network effects model, with 'porting' playing a role analogous to 'converters' in the simpler direct network effects models. With 'porting', software developed for one platform can be converted to run on another (at a cost lower than that of direct production). We examined general properties of this model, looking, in particular, at what occurs when one (dominant) platform is controlled by a single firm, the Monopolist, who is able to control the cost of porting to a competitor platform (at the cost of some expenditure on the Monopolist's part). We demonstrated the existence of a platform (and porting) equilibrium and examined various associated properties, such as the discontinuity in the monopolist's demand function.

Next we turned to the question of consumer and social welfare. It was shown that, the effect on welfare both of monopoly pricing and higher porting costs depended crucially on the degree of diminishing returns to platform size in the indirect network effects function $(\nu)$. If diminishing returns were weak then monopoly pricing had a negative effect on welfare but the effect of the higher porting costs was ambiguous, while with strongly diminishing returns the converse held, that is the effect of monopoly pricing was ambiguous but higher porting costs had a negative effect.

Finally, we provided an illustrative example using a specific case of our model. We showed that, in this example, the social and consumer welfare losses arising from the control of porting combined with monopoly pricing dwarfed the welfare effects stemming from monopoly pricing alone. In particular, consumer welfare losses from the combination of higher porting costs and higher prices were over nine times higher than those arising from higher prices alone. For total welfare, there was almost no effect of monopoly pricing alone but a significant reduction when the monopolist controlled both prices and porting costs (in this second case the welfare loss was equal to approximately three fifths of the monopolist's profits). Of course this is a single example and without either calibrating

\footnotetext{
${ }^{28}$ In fact it would no doubt be possible to choose a model such that the ability to control porting increased welfare - all one would need is for the benefits of platform 'standardization' to be sufficiently strong.
} 
from empirical data or extensive robustness-checking one would not wish to use the results for policy-making. Nevertheless, it does provide a useful example that helps put flesh on the dry bones of the general model.

These results, taken together, have important consequences for competition policy. They demonstrate how, in a two-sided market environment, anti-competitive behaviour may manifest indirectly through actions taken to control porting rather than through direct tying or pricing behaviour. Furthermore, for the monopolist the benefits of controlling porting may also accrue indirectly: that is, by increasing the prices that can be charged at a given level of demand rather than increasing demand. Returning to the examples discussed in the introduction, we would suggest that an analysis based on the control of porting provides a better way of understanding the effects and motivations of a dominant firm than alternative approaches, such as those based on traditional theories of tying or even switching costs. ${ }^{29}$

Of course from an antitrust point of view this is not enough - simply establishing a potential 'anti-competitive' motivation for a firms behaviour is not sufficient to show such actions will actually harm welfare. In this regard, as already mentioned, our central result was that the crucial parameter to estimate is the curvature of the indirect network effects function (that is the degree of diminishing returns to platform size). When the degree of diminishing returns is high - the benefit of a millionth user is much less than the thousandth - the control of porting unambiguously harms welfare but when the degree of diminishing returns is low - the benefit of the millionth user and the thousandth user is similar - then the control of porting has an ambiguous impact (it may even increase welfare). Given this, the first step for an antitrust economist tasked with analyzing the control of porting in a particular industry would be to estimate the form of the indirect network effects function for the particular platforms under consideration. ${ }^{30}$

When the control of porting does harm welfare, policy-makers may wish to take steps to reduce the control of porting by a dominant firm. One simple way to do this is to promote

\footnotetext{
${ }^{29}$ Though, of course, in one sense the control of porting can be seen as a special case of tying (or the creation of switching cost) in which the 'tie' is not aimed at competing providers of the tied good but at the owners of competing platforms.

${ }^{30}$ As mentioned in an earlier footnote there is little empirical evidence for the form of 'network effects' (whether in two-sided markets or traditional 'network' industries). The fact that most of the models in the theoretical literature use a linear specification is due solely to analytical tractability and not to any empirical support for this functional form - a choice which, as this paper has shown, is not an innocent one.
} 
'open standards' at the interface between the 'software/service' and the platform. For example, in the case of TPMs/DRMs (Technological Protection Measures/Digital Rights Management) systems a policy-maker could promote (or require) interoperability between different TPM/DRM systems so that the music ('software' in our terminology) purchased from any given vendor will work on any given digital music player (the platform). ${ }^{31}$

Similarly, in the case of the EU dispute with Microsoft over Microsoft's Windows Media Player, rather than requiring unbundling the authorities could simply require that any audio formats specific to Windows Media Player must be 'open' and freely licensable so as to ensure that it is easy to port music and complementary services to a media player on another platform such as Linux. The same approach would also apply to web browsers where there already exist an extensive set of open standards developed by the W3C. Again, rather than requiring Microsoft to unbundle Internet Explorer the authorities could simply press for 'standards-compatibility'. In this way developers of websites and other forms of web-services would be able to develop in a platform-neutral way (essentially the cost of porting to a different platform such as Linux+Firefox would then be zero) with all the associated long-run benefits for competition and consumer choice.

Finally, we mention some of potential avenues for future work. One of the most obvious improvements that could be made would be to replace the simple monopoly model with an oligopoly in which each platform has a profit-maximizing owner. Porting, and the manner in which it may be controlled, have been modelled in a fairly simple manner. One might improve this in various ways. For example, one could change from a 'black box' cost function $e$ to a setup where $f_{A}$ increases with $f^{p}$ - this would correspond to an 'obfuscation' situation where increasing porting costs to competitor platforms also increases the cost of producing software on one's own platform.

One could also add dynamics to the model (though this would also greatly increase complexity). For example, rather than having a fixed static demand one could allow consumers to arrive over time. ${ }^{32}$ Alternatively consumers could make repeat purchases but with a switching cost if a different platform were chosen in a subsequent period.

\footnotetext{
${ }^{31}$ At the present time this very issue of DRM interoperability is being debated both at the EU level and in various individual European countries in relation to Apple's FairPlay DRM.

${ }^{32}$ See, for example, Cabral (2007) or the model of Fudenberg and Tirole (2000) which gives rise to limitpricing behaviour on the part of the monopolist. Though we note that the addition of dynamics adds very substantial technical complexity.
} 
Finally, it would be interesting to explore the consequences of allowing for innovation in software provision perhaps via the introduction of a quality ladder. Such an approach would raise additional thorny questions about the welfare impact of monopolist behaviour if innovation were not barrier to entry neutral. For example, if innovations while increasing quality also made it easier to port from one platform to another (consider the case of Java or the emergence of the web and web browsers as a fully-fledged application development platform). ${ }^{33}$ In this case, efforts to obstruct porting would also hinder innovation, with all the attendant consequences for welfare.

\section{A. Proofs}

A.1. Proof of Lemma 2. Recall that the conditional utility advantage of platform A over platform $\mathrm{B}$ for consumer $t$ when platform size is $n_{A}$ :

$$
\hat{A}\left(t, n_{A}\right)=u_{A}\left(t, n_{A}\right)-u_{B}\left(t, 1-n_{A}\right)
$$

and the utility advantage (function), which gives the utility advantage of platform A over $\mathrm{B}$ if $t$ is the marginal consumer (so $\left.t=n_{A}\right)$ :

$$
A(t)=\hat{A}(t, t)
$$

Suppressing $n_{A}$ for the time being we shall simply write $\hat{A}(t)$.

Since 'heterogeneity cost' for a consumer is increasing in the distance of the consumer from the chosen platform we have that $\forall t, \hat{A}^{\prime}(t)<0$. Then $\hat{A}\left(t_{m}\right)>0$ implies $\hat{A}(t)>$ $0, \forall t \leq t_{m}$. Conversely if $\hat{A}\left(t_{m}\right)<0$ then $\hat{A}(t)<0 \forall t \geq t_{m}$.

Now a consumer (with expectations of platform A size equal to $n_{A}$ ) chooses platform A over $\mathrm{B}$ iff $\hat{A}(t) \geq 0$. Thus if a consumer with index $t_{m}$ chooses platform $\mathrm{A}$ then all consumers with index $t \in\left[0, t_{m}\right]$ choose platform A. Similarly if a consumer with index $t_{m}$ chooses platform $\mathrm{B}$ then all consumers with index $t \in\left(t_{m}, 1\right]$ choose platform $\mathrm{B}$.

In particular this immediately implies that if there exists $t_{m} \in[0,1], \hat{A}\left(t_{m}\right)=0$ (and there is at most one such solution since $\hat{A}^{\prime}<0$ ) then this is the marginal consumer and the resulting platform size of $\mathrm{A}$ is $t_{m}$. This is because for $t \in\left[0, t_{m}\right], \hat{A}(t)>0$ so these

\footnotetext{
${ }^{33}$ See e.g. Farrell and Katz (2000) on network monopolies and downstream innovation.
} 
consumers choose platform A while for $t \in\left(t_{m}, 1\right], \hat{A}(t)<0$ so these consumers choose platform B.

For the extremal cases by the same arguments if $\hat{A}(0)<0$ then all consumers choose platform B and if $\hat{A}(1)>0$ then all consumer's choose platform A.

Furthermore, only one of these alternatives is possible so there is a unique implied platform size for any given assumed $n_{A}$. Thus one may define a function $f:[0,1] \rightarrow[0,1]$ where for a given assumed platform size, $n, f(n)$ is the resulting implied platform size.

Imposing rational expectations then implies that $n_{A}$ is an equilibrium if and only if $n_{A}$ is a fixed point of $f$. But $n_{A}$ is a solution of $f(n)=n \Leftrightarrow n_{A} \in E$. QED

Remark: Equilibria $t \in E_{-0}$ are often termed standardization or tipping equilibria as they involve all consumers joining a single platform.

Remark: This result sets up an implicit equivalence between platform size and the marginal consumer (where the term marginal is broadened to include the tipping situations where $t_{m}=0$ or 1 and $A\left(t_{m}\right) \neq 0$

Stability of Equilibria: Suppose we have equilibrium $t_{m} \in E_{0}$ with $A^{\prime}\left(t_{m}\right)<0$. Suppose that there is a perturbation in expectations so that a platform size of $t_{m}+\epsilon$ is expected instead of $t_{m}$ (where $\epsilon>0$ ). Since $A^{\prime}<0$ we must have $\hat{A}\left(t_{m}+\epsilon, t_{m}+\epsilon\right)=$ $A\left(t_{m}+\epsilon\right)<0$. Now in the interior all functions are continuous so $\hat{A}$ is continuous. Thus $\delta$ in the region $t_{m}+\epsilon$ we have that $\hat{A}\left(x, t_{m}+\epsilon\right)<0$ for $x \in\left(t_{m}+\epsilon-\delta, t_{m}+\epsilon\right]$. But then all consumers with indices in that range wish to leave platform A and go to platform B. Repeating this process we converge back to the equilibrium $t_{m}$. The analogous argument for negative $\epsilon$ shows the equilibrium is stable to perturbation downwards in expectations. Thus the equilibrium is stable.

The exact same form of argument applied to an equilibrium $t_{m} \in E_{-0}$ shows that it too is stable. QED.

A.2. Proof of Lemma 3 (Porting Lemma). The result will follow from two claims: Claim 1: Suppose that a platform has a piece of software produced directly for it. Then $s_{X}, p_{X}^{s}$ are determined by $f_{X}^{d}$ (the direct cost of software production) alone. We may therefore take $f_{X}=f_{X}^{d}$ in all the formulas obtained above (it is immaterial for the purposes of calculating all equilibrium values whether software is ported or produced directly for this platform). 
Proof. The cost of porting is less than the cost of direct production. Thus as long as one software firm enters directly it must be the profit condition of that firm that binds (i.e. is zero). This condition alone determines the total number of software firms and software prices.

Clearly if no firm produces directly there can be no porting as there would be nothing to port.

Claim 2: If porting is possible in both directions and both platforms have some software produced directly then both platforms have the same amount of software produced for them. This in turn implies $f_{A}^{d} n_{B}=n_{A} f_{B}^{d}$.

Proof. If software is produced directly then all software that could have ported must have been (since it is cheaper to port). Let $d, p\left(d^{\prime}, p^{\prime}\right)$ be the amount of directly produced software and ported software respectively on A (B). Then $s_{A}=d+p$ but $p^{\prime}=d, p=d^{\prime}$ so $s_{A}=s_{B}$. If this is the case it requires $f_{A}^{d} n_{B}=n_{A} f_{B}^{d}$ since $s_{X}^{2} f_{X}^{d}=n_{X}$.

The statement of the Lemma specifically excludes the possibility that $f_{A}^{d} n_{B}=n_{A} f_{B}^{d}$. This immediately implies the converse of the claim, namely that that software is produced directly for at most one platform. The Lemma is proved. QED.

A.3. Proof of Lemma 5. Proof of existence: Fix an equilibrium $t_{e}^{0} \in E_{0}\left(p_{A}^{0}, \ldots\right)$ then we can define $t_{e}\left(p_{A}, \ldots\right)$ by picking $t_{e} \in E\left(p_{A}, \ldots\right)$ consistent with $t_{e}^{0}$. Since $A(t)$ is continuously differentiable so too will be $t_{e}\left(p_{A}, \ldots\right)$ (at least almost everywhere - see below). For notational convenience whenever a parameter is fixed we shall drop it from the list of arguments to $t, A, \ldots$.

Differentials: implicitly differentiate the equation $A(t)=0$ with respect to the relevant variable $\left(p_{A}, f_{A}, f_{B}\right)$. Since increasing A's price by $d p$ shifts the $A(t)$ curve down by $d p$ reducing $t_{e}$ the sign of the differential is as stated. Similarly increasing $f_{A}$ shifts the platform advantage curve down and therefore the advantage curve down reducing $t_{e}$ and therefore the differential with respect to $f_{A}$ must be negative (and conversely for $f_{B}$ ).

Remarks on discontinuity and profit maximization: Fix $f_{A}, f_{B}$, then $t_{e}\left(p_{A}\right)=$ $A^{-1}\left(p_{A}\right)$ is the demand function faced by M. From the previous result we know this is downward sloping. Now take a stable equilibrium $t^{0}$ when $p_{A}=0$ and assume there exists an adjacent non-extremal equilibrium $t^{0^{\prime}} \leq t^{0}$ (which must be unstable). Then there 
must exist a maximum of $A(t)$ at $t^{1} \in\left(t^{0^{\prime}}, t^{0}\right)$ with $A^{\prime}\left(t^{1}\right)=0$ and the demand function $t_{e}\left(p_{A}\right)\left(t_{e}(0)=t^{0}\right)$ is discontinuous at $t^{1}$ with $p_{A}^{d}=A\left(t^{1}\right)$.

Despite this there will still exist a profit maximizing price $p_{A}^{d}>p_{A}^{m}$ since

$$
\lim _{t \rightarrow t_{+}^{1}} A^{-1}(t)=-\infty
$$

A.4. Proof of Lemma 6. Set all of M's control variables to their initial value. Suppose first there are no discontinuities in M's demand function. This occurs iff there exists no zeroes of $A^{\prime}(t)$, i.e iff $A(t)$ is monotonic. Since we assume existence of a stable interior equilibria must have that $A(t)$ is downward sloping. Thus we have a downward sloping demand function. This gives a well-defined and continuous profit function on a compact set (demand space extends only from 0 to 1 ). Thus the profit function has a maximum which it attains somewhere on the set. QED.

Again, set all of M's control variables to their initial value. So assume that there there is a discontinuity in the demand function, i.e. that there exists a $t$ with $A^{\prime}(t)=0$. Pick an interior stable equilibrium. Then by Lemma 5 there exists an associated welldefined demand function. Furthermore, there exists locally a unique profit-maximizing price which occurs prior to any discontinuous jump (downwards) in the demand function. But this ensures the existence of equilibrium in the overall game since it means that at any discontinuity in the demand function the profit function is downwards sloping. QED.

A.5. Proof of Welfare-Related Propositions. Consumer welfare as a function of platform A's size (t) is given by (for simplicity $\phi$ is omitted):

$$
W^{C}(t)=-t \cdot p_{A}+t \nu_{A}(t)+(1-t) \nu_{B}(1-t)-\int_{0}^{t} h_{A}(x) d x-\int_{t}^{1} h_{B}(x) d x
$$

Moving to total welfare we need only add in the relevant expression for $\Pi_{A}=t \cdot p_{A}-$ $e\left(f^{p}\right)$. Thus:

$$
W=t \cdot p_{A}-e\left(f^{p}\right)-t \cdot p_{A}+t \nu_{A}(t)+(1-t) \nu_{b}(1-t)-\int_{0}^{t} h_{A}(t) d t-\int_{t}^{1} h_{B}(t) d t
$$

A.5.1. Proof of Lemma 7. Differentiating consumer welfare with respect to $t$ yields:

$$
\frac{d W^{C}}{d t}=-p_{A}+\nu_{A}(t)-\nu_{B}(1-t)-h_{A}(t)+h_{B}(1-t)+t \nu_{A}^{\prime}(t)-(1-t) \nu_{A}^{\prime}(1-t)
$$


This simplifies to $(A(t)$ is the utility advantage of A over B defined previously):

$$
\frac{d W^{C}}{d t}=A(t)+t \nu_{A}^{\prime}(t)-(1-t) \nu_{B}^{\prime}(1-t)=A(t)+\mu(t)
$$

where we have defined:

$$
\mu(t)=t \nu_{A}^{\prime}(t)-(1-t) \nu_{B}^{\prime}(1-t)
$$

At an equilibrium $t_{e}, A\left(t_{e}\right)=0$, so this reduces to:

$$
\frac{d W^{C}}{d x}=\mu\left(t_{e}\right)
$$

QED.

\section{A.5.2. Proof of Lemma 8.}

$$
\frac{d W^{C}}{d p_{A}}=-t+\frac{d t}{d p_{A}} \frac{d W^{C}}{d t}
$$

Considered at an asymmetric equilibrium the second term will be greater than or less than zero depending on whether $\mu$ is less than or greater than zero. If $\mu$ is non-negative then the second term is negative and total sum will be negative. If $\mu$ is negative the total sum will be ambiguous (depending on the relative magnitudes of the two terms). Thus, if network effects do not show very strong diminishing returns (and so $\mu$ is nonnegative) welfare changes negatively with increasing price. If $\mu$ is negative (as it would in the circular city model) then the effect on consumer welfare depends on the relative size of the monopoly pricing costs (first term) versus the network externality (second term).

Turning to total welfare we have:

$$
\frac{d W}{d p_{A}}=\frac{d \Pi_{A}}{d p_{A}}+\frac{d W^{C}}{d p_{A}}=\frac{d t}{d p_{A}}\left(p_{A}+\frac{d W^{C}}{d t}\right)
$$

The term outside the brackets is negative but again here the second term can have either positive or negative sign in general. NB: when the monopolist is profit maximizing the differential of monopolist profits with respect to price is zero. Thus, the differential of total welfare equals the differential of consumer welfare.

A.5.3. Proof of Lemma 9. The change in consumer welfare as a consequence of an increase in the cost of porting is: 


$$
\frac{d W^{C}}{d f^{p}}=(1-t) \frac{d \nu_{B}}{d f^{p}}+\frac{d t}{d f^{p}} \frac{d W^{C}}{d t}
$$

The first term is clearly negative since software provision on platform B declines as porting costs go up. The analysis of the second term is similar to the case of a change in price. As platform A's market share increases as porting costs increase the second term will be greater than or less than zero depending on whether $\mu$ is greater than or less than zero. Thus, if $\mu$ is less than zero (strongly diminishing marginal network effects) the total will be unambiguously negative and consumer welfare declines with increases in porting costs. If $\mu$ is positive then the total has ambiguous sign in general, and will depend on relative sizes of the two terms.

For total welfare we have:

$$
\frac{d W}{d f^{p}}=\frac{d \Pi_{A}}{d f^{p}}+\frac{d W^{C}}{d f^{p}}
$$

When profit-maximizing the first term is zero and the differential of total welfare equals that of consumer welfare. When not at a profit-maximizing level of porting costs the first term is positive. In this case whether the total is positive or negative will depend on the specific circumstances.

A.6. Proof of Lemma 10. The stated results all follow by straightforward examination of changes in the sizes of the various terms in the proof of Lemma 9 above.

\section{B. Software Production}

There are two main methods of modelling product variety in the literature. One based on monopolistic competition and one based on locational models. The monopolistic competition approach has already been extensively used to demonstrate indirect network effects in hardware/software systems (see e.g. Church and Gandal (1992); Church, Gandal, and Krause (2003)). One can also use an approach based on locational differentiation and that is the approach we adopt here.

Software firms on platform $\mathrm{X}$ have fixed $\operatorname{costs} f_{X}$ and marginal $\operatorname{costs} c_{X}^{s}$. Marginal costs are assumed to be constant across the two platforms but fixed costs are not. Because software production involves a fixed cost it cannot be provided competitively. Instead we introduce a locational model of product differentiation and imperfect competition 
For each platform, software 'space' is represented as a circle (of circumference 1). Software firms are assumed to locate symmetrically (and therefore equidistantly) in this space. $^{34}$ while consumers are distributed uniformly over it (so total demand for software on platform $\mathrm{X}$ is the total number of consumers on that platform: $n_{X}$ ). Following the standard circular city model ${ }^{35}$ we have consumer's (expected) utility from software consumption is:

$$
u_{X}^{s}\left(s_{X}, p_{X}^{s}\right)=-E\left[d\left(x\left(s_{X}\right)\right)\right]-p_{X}^{s}
$$

Where $d$ is a 'travel' cost function of all locational models, $x\left(s_{X}\right)$ is the distance a consumer is from the nearest software, and $E$ is the expectation operator. Average travel cost is used because it is assumed that consumers make their decision when they do not yet know their exact position in software space relative to software producers. Thus they base their decisions on expected costs (which will be common across consumers). We shall assume a linear travel cost, $d(x)=k x$.

B.1. Solving. The main result can be stated in the form of a lemma:

Lemma 11. Given expected platform sizes $n_{X}^{e}$ the equilibrium level of software production, associated prices, and software utility are:

$$
\begin{gathered}
s_{X}=\sqrt{\frac{k n_{X}^{e}}{f_{X}}} \\
p_{X}=c_{X}^{s}+\sqrt{\frac{k f_{X}}{n_{X}^{e}}} \\
u_{X}^{s}\left(s_{X}, p_{X}^{s}\right)=-c_{X}^{s}-\frac{5}{4} \sqrt{\frac{k f_{X}}{n_{X}^{e}}}
\end{gathered}
$$

Proof. The setup is exactly the same as the textbook circular city model (see e.g. Tirole 1988) except that demand rather than being 1 is equal to the expected market size of that platform: $n_{X}^{e}$. This leaves prices unchanged (since the shape of demand curve is unchanged), so in equilibrium: $p_{X}=c_{X}^{s}+\frac{k}{s_{X}}$ where $k$ is the cost of travel $(d(x)=k x)$.

\footnotetext{
${ }^{34}$ Firms' location decisions could be endogenized and this outcome derived as an equilibrium configuration - see Economides (1989) However we choose to take this as an assumption for the sake of simplicity.

${ }^{35}$ See e.g. Tirole (1988) for details.
} 
Firms locate equidistantly and each face the same level of demand equal to total demand divided by the number of software firms. To determine the number of software firms we use the free entry condition which means that in equilibrium firms earn zero net profits i.e. they cover fixed costs:

$$
\left(p_{X}-c_{X}^{s}\right) \frac{n_{X}^{e}}{s_{X}}-f=0 \Rightarrow \frac{k n_{X}^{e}}{s_{X}^{2}}-f=0 \Rightarrow s_{X}=\sqrt{\frac{k n_{X}^{e}}{f}}
$$

This in turn gives:

$$
p_{X}=c_{X}^{s}+\sqrt{\frac{k f}{n_{X}^{e}}}
$$

The form of the software utility functions in our particular case? Consumers do not know the exact location of firms in advance so they base their decisions on the expected distance from a software producer. Software firms locate randomly but equidistantly on the circle and consumers are uniformly distributed thus expected distance between a consumer and the nearest software is a quarter of the distance between firms. Distance between firms is the inverse of the number of firms, $s_{X}$. We therefore have:

$$
u_{X}^{s}\left(s_{X}, p_{X}^{s}\right)=-p_{X}^{s}-k\left(\frac{1}{4 s_{X}}\right)
$$

Substituting the values for $p_{X}, s_{X}$ we have ${ }^{36}$ :

$$
u_{X}^{s}\left(s_{X}, p_{X}^{s}\right)=-c_{X}^{s}-\frac{5}{4} \sqrt{\frac{k f}{n_{X}^{e}}}
$$

Remark: Since the constant $\frac{5 \sqrt{k}}{4}$ can be absorbed into fixed cost $f_{X}$ this variable will be omitted in future and we have:

$$
u_{X}^{s}\left(s_{X}, p_{X}^{s}\right)=-c_{X}^{s}-\sqrt{\frac{f}{n_{X}^{e}}}
$$

We can now substitute this expression for $u_{X}^{s}$ to obtain:

Corollary 12. The reduced form of the utility function is:

\footnotetext{
${ }^{36}$ The result for the quadratic distance case would be: $u_{X}^{s}\left(s_{X}, p_{X}^{s}\right)=-c_{X}^{s}-\sqrt{\frac{k f}{n_{X}^{e}}}-\frac{f}{16 n_{X}^{e}}$
} 


$$
u_{X}(t)=\phi-p-h_{X}(t)-c_{X}^{s}-\sqrt{\frac{f_{X}}{n_{X}^{e}}}
$$

Remark: Note how this shows that the model displays indirect network effects as the reduced form expression for utility (from 'software') displays positive feedback between the total number of consumers on $\mathrm{X}$ and the utility of an individual on $\mathrm{X}: \frac{d}{d n_{X}^{e}} u_{X}^{s^{\prime}}>0$.

\section{REFERENCES}

Mark Armstrong. Competition in Two-Sided Markets. Technical report, 2005. Forthcoming in the Rand Journal of Economics (December 2006).

Mark Armstrong and Julian Wright. Two-sided Markets, Competitive Bottlenecks and Exclusive Contracts. Technical report, 2005. Forthcoming in Economic Theory.

B. Douglas Bernheim and Michael D Whinston. Exclusive Dealing. The Journal of Political Economy, 106(1):64-103, February 1998. ISSN 00223808.

T. Bresnahan. The Economics of The Microsoft Case, 2001. Unpublished discussion paper. Luis Cabral. Dynamic Price Competition with Network Effects. Technical report, 2007. Unpublished.

Dennis W. Carlton and Michael Waldman. The Strategic Use of Tying to Preserve and Create Market Power in Evolving Industries. RAND Journal of Economics, 33(2):194220, Summer 2002.

Jay-Pil Choi. The Provision of (Two-Way) Converters in the Transition Process to a New Incompatible Technology. Journal of Industrial Economics, 45(2):139-153, 1997.

Jay Pil Choi. Tying in Two-Sided Markets with Multi-Homing. Working Papers 06-04, NET Institute, September 2006.

Jeffrey Church and Neil Gandal. Network Effects, Software Provision and Standardization. Journal of Industrial Economics, 40(1):85-103, 1992.

Jeffrey Church, Neil Gandal, and David Krause. Indirect Network Effects and Adoption Externalities, 2003. mimeo.

Stephen Davis and Kevin Murphy. A Competitive Perspective on Internet Explorer. AER Papers and Proceedings, 90(2):184-187, 2000.

Nicholas Economides. Symmetric Equilibrium Existence and Optimality in Differentiated Products Markets. Journal of Economic Theory, 47(1):178-194, 1989. 
Joseph Farrell and Michael Katz. Innovation, Rent Extraction, and Integration in Systems Markets. Journal of Industrial Economics, 48(4):413-432, 2000.

Joseph Farrell and G. Saloner. Converters, Compatibility, and the Control of Interfaces. Journal of Industrial Economics, 40(1):9-35, 1992.

Franklin Fisher. The IBM and Microsoft Cases: What's the Difference. AER Papers and Proceedings, 90(2):180-183, 52000.

D. Fudenberg and J. Tirole. Pricing a Network Good to Deter Entry. Journal of Industrial Economics, 48(4):373-90, 2000.

R. Gilbert and Michael Katz. An Economist's Guide to U.S. v. Microsoft. JEP, 15(2): 25-44, 2001.

Richard J. Gilbert and Michael H. Riordan. Product Improvement and Technological Tying in a Winner-Take-All Market. Journal of Industrial Economics, 55(1):113-139, 032007.

C. Hall and R. Hall. Toward a Quantification of the Effects of Microsoft's Conduct. AER Papers and Proceedings, 90(2):188-191, 52000.

R. Jackson. Findings of Fact in the case of United States vs. Microsoft, 111999.

Benjamin Klein. The Microsoft Case: What Can a Dominant Firm Do to Defend Its Market Position? JEP, 15(2):45-62, 2001.

S. Liebowitz and S. Margolis. Winner, Losers and Microsoft: Competition And Antitrust in High Technology. Independent Institute, 1999.

Andrew Odlyzko and Benjamin Tilly. A refutation of Metcalf's Law and a better estimate for the value of networks and network interconnections, 2005.

Jean-Charles Rochet and Jean Tirole. Platform Competition in Two-Sided Markets. Journal of the European Economic Association, 1(4):990-1029, 062003.

Jean-Charles Rochet and Jean Tirole. Two-Sided Markets : A Progress Report. IDEI Working Papers 275, Institut d'conomie Industrielle (IDEI), Toulouse, November 2005. Forthcoming in the RAND Journal of Economics.

G. M. Peter Swann. The Functional Form of Network Effects. Information Economics and Policy, 14(3):417-429, September 2002.

M. Whinston. Tying, Foreclosure, and Exclusion. American Economic Review, 80(4): 837-859, 1990. 
M. Whinston. Exclusivity and Tying in U.S. v. Microsoft: What We Know, and Don't Know. JEP, 15(2):63-80, 2001. 